\title{
Severe hypokalaemia in diabetic ketoacidosis: a contributor to central pontine myelinolysis?
}

\author{
A Chinoy ${ }^{1,2}$, N B Wright ${ }^{3}$, M Bone $^{4}$ and R Padidela1,2 \\ 1Department of Paediatric Endocrinology, Royal Manchester Children's Hospital, Manchester, UK, 2Faculty of Biology, \\ Medicine and Health, University of Manchester, Manchester, UK, ${ }^{3}$ Department of Paediatric Radiology, Royal \\ Manchester Children's Hospital, Manchester, UK, and 4Department of General Paediatrics, Royal Manchester \\ Children's Hospital, Manchester, UK
}

Correspondence should be addressed to A Chinoy

Email

amishchinoy@doctors.org.uk

\begin{abstract}
Summary
Hypokalaemia at presentation of diabetic ketoacidosis is uncommon as insulin deficiency and metabolic acidosis shifts potassium extracellularly. However, hypokalaemia is a recognised complication of the management of diabetic ketoacidosis as insulin administration and correction of metabolic acidosis shifts potassium intracellularly. We describe the case of a 9-year-old girl with newly diagnosed type 1 diabetes mellitus presenting in diabetic ketoacidosis, with severe hypokalaemia at presentation due to severe and prolonged emesis. After commencing management for her diabetic ketoacidosis, her serum sodium and osmolality increased rapidly. However, despite maximal potassium concentrations running through peripheral access, and multiple intravenous potassium 'corrections', her hypokalaemia persisted. Seventy two hours after presentation, she became drowsy and confused, with imaging demonstrating central pontine myelinolysis - a rare entity seldom seen in diabetic ketoacidosis management in children despite rapid shifts in serum sodium and osmolality. We review the literature associating central pontine myelinolysis with hypokalaemia and hypothesise as to how the hypokalaemia may have contributed to the development of central pontine myelinolysis. We also recommend an approach to the management of a child in diabetic ketoacidosis with hypokalaemia at presentation.
\end{abstract}

\section{Learning points:}

- Hypokalaemia is a recognised complication of treatment of paediatric diabetic ketoacidosis that should be aggressively managed to prevent acute complications.

- Central pontine myelinolysis is rare in children, and usually observed in the presence of rapid correction of hyponatraemia. However, there is observational evidence of an association between hypokalaemia and central pontine myelinolysis, potentially by priming the endothelial cell membrane to injury by lesser fluctuations in osmotic pressure.

- Consider central pontine myelinolysis as a complication of the management of paediatric diabetic ketoacidosis in the presence of relevant symptoms with profound hypokalaemia and/or fluctuations in serum sodium levels.

- We have suggested an approach to the management strategies of hypokalaemia in paediatric diabetic ketoacidosis which includes oral potassium supplements if tolerated, minimising the duration and the rate of insulin infusion and increasing the concentration of potassium intravenously (via central line if necessary).

\section{Background}

Children with type 1 diabetes mellitus often present in diabetic ketoacidosis (DKA). DKA causes varying degrees of derangement of fluid balance and electrolytes due to hyperglycaemia-induced osmotic fluid shifts, osmotic diuresis, complications of end-organ injury and therapies used in its management. It is therefore not surprising that 
numerous international guidelines have been developed to prevent morbidity and mortality associated with management of DKA $(1,2)$.

Osmotic diuresis, secondary hyperaldosteronism and emesis in DKA all cause net depletion of body stores of potassium (1). Despite this, hypokalaemia is not commonly encountered at presentation in DKA because insulin deficiency and metabolic acidosis shifts potassium from the intracellular to the extracellular compartment (3). However, hypokalaemia is a recognised complication of the management of DKA and occurs due to administration of insulin and correction of metabolic acidosis, which drives potassium ions intracellularly (1).

Central pontine myelinolysis (CPM) is a noninflammatory demyelinating disorder of the pons, though extra-pontine sites can also be affected; it is very rarely seen in the paediatric population. Although the precise pathophysiology is unclear, it is thought to be driven by fluctuating osmotic levels, with rapid correction of hyponatraemia by far the commonest risk factor (4).

Here, we report a child with newly diagnosed diabetes presenting in DKA, with marked hypokalaemia and serum sodium at the lower end of the normal range at presentation. Serum sodium increased quickly after initiation of treatment; however, hypokalaemia was refractory to treatment. Unfortunately, later during the admission she progressed to develop CPM.

\section{Case presentation}

A nine-year-old girl, who had been previously fit and well, presented to the children's emergency department with a 3-week history of persistent and recurrent vomiting, abdominal pain, poor oral intake and weight loss. DKA was confirmed when blood results revealed: $\mathrm{pH} 7.19$ (normal range 7.35-7.45), blood glucose $17.9 \mathrm{mmol} / \mathrm{L}$ (normal range $4-7 \mathrm{mmol} / \mathrm{L}$ ), bedside ketones $>7 \mathrm{mmol} / \mathrm{L}$ (normal $<0.1 \mathrm{mmol} / \mathrm{L}$ ), bicarbonate $4.6 \mathrm{mmol} / \mathrm{L}$ (normal range $22-28 \mathrm{mmol} / \mathrm{L}$ ). Notably, at presentation her serum sodium was $133 \mathrm{mmol} / \mathrm{l}$ (normal range $133-146 \mathrm{mmol} / \mathrm{L}$ ), but her serum potassium was markedly low at $2.3 \mathrm{mmol} / \mathrm{L}$ (normal range $3.5-5.0 \mathrm{mmol} / \mathrm{L}$ ). Calculated serum osmolality was $290 \mathrm{mosm} / \mathrm{kg}$.

Management was initiated as per national guidelines (2): intravenous fluids initiated at maintenance rate plus 5\% fluid deficit to be corrected over $48 \mathrm{~h}$, with fluid containing $40 \mathrm{mmol} / \mathrm{L}$ of potassium chloride; insulin was commenced $1 \mathrm{~h}$ later at 0.05 units $/ \mathrm{kg} / \mathrm{h}$. Her blood glucose reduced to $5.2 \mathrm{mmol} / \mathrm{L}$ over the first $4 \mathrm{~h}$ of admission, and thereafter largely remained between 4 and $8 \mathrm{mmol} / \mathrm{L}$ over the next few days. Her serum sodium increased from 133 to $148 \mathrm{mmol} / \mathrm{L}$ over the first $12 \mathrm{~h}$ of admission, and with it the calculated serum osmolality also increased from 290 to $301 \mathrm{mosm} / \mathrm{kg}$. The serum sodium then remained at this concentration over the next few days. Over the first $48 \mathrm{~h}$, her ketoacidosis improved, however, her hypokalaemia persisted. Her vomiting meant she could not tolerate oral potassium supplements. Despite ten intravenous 'corrections' of potassium chloride (each correction diluted in $5 \mathrm{~mL} / \mathrm{kg}$ of $0.9 \%$ sodium chloride, amounting to approximately $2 \mathrm{mmol} / \mathrm{kg}$ of extra potassium chloride; total extra fluid volume equivalent to $50 \mathrm{~mL} / \mathrm{kg}$ ), her serum potassium remained largely $2.0-3.0 \mathrm{mmol} / \mathrm{L}$ throughout.

On day four of admission, she suddenly became sleepy, with slow and vague responses, and a Glasgow Coma Score fluctuating between 13 and 15. At the same time, new onset generalised hyper-reflexia was noted.

\section{Investigation}

An urgent CT scan demonstrated features of CPM (Fig. 1A), which was confirmed on MRI scan the following day (Fig. 1B). Her potassium at the time was $2.8 \mathrm{mmol} / \mathrm{L}$, sodium was $146 \mathrm{mmol} / \mathrm{L}$, blood glucose was $5.9 \mathrm{mmol} / \mathrm{L}$ and calculated serum osmolality was $304 \mathrm{mosm} / \mathrm{kg}$.

\section{Outcome and follow-up}

Her hypokalaemia improved over the next $48 \mathrm{~h}$, as her vomiting settled and she started tolerating enteral feeds. She remained an in-patient for a further month for neurorehabilitation in view of her dysphagia, dysarthria and
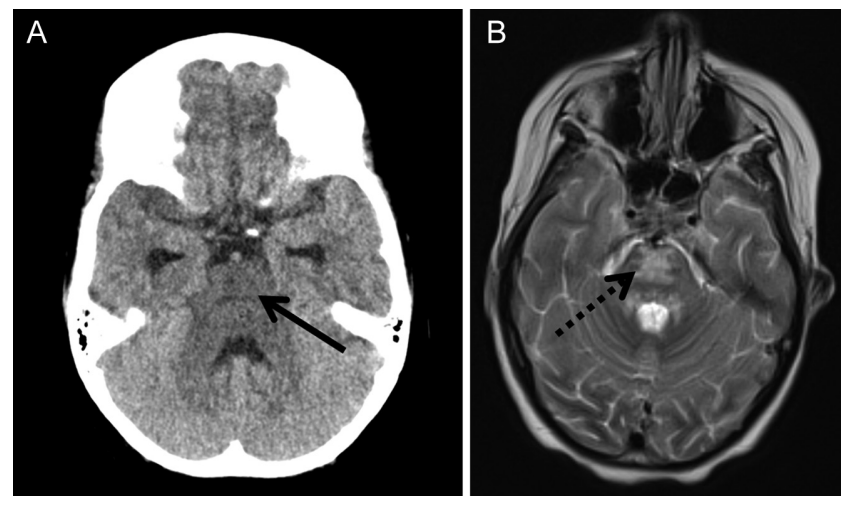

\section{Figure 1}

(A) Transverse image from non-enhanced CT scan demonstrating generalised hypodensity in the pontine area (solid arrow), suggestive of central pontine myelinolysis. (B) Transverse image from T2-weighted MRI scan demonstrating abnormal high T2 signal in the pons (dotted arrow), confirming central pontine myelinolysis. 
motor deficit. With intensive physiotherapy, occupational therapy and speech and language therapy, both within the hospital and within the community, she has shown improvement in her neurological function with only a mild left lower limb evident 1 year after the event.

Her clinical progress, charted in terms of her serum sodium and potassium concentration over the first week of her admission, has been shown in Fig. 2 .

\section{Discussion}

Profound hypokalaemia is associated with muscle necrosis and even ascending paralysis. Furthermore, it can cause electrophysiological changes resulting in atrial and ventricular arrhythmias. Therefore, unsurprisingly, guidelines on the management of DKA stress the importance of preventing hypokalaemia with provision of adequate potassium chloride in intravenous fluids and to correct any evolving hypokalaemia promptly $(1,2)$. Furthermore, international guidelines recommend minimising the time on intravenous insulin infusion and the dose, as this clearly exacerbates hypokalaemia, as well as increasing the rate of intravenous potassium replacement up to a maximum of $0.5 \mathrm{mmol} / \mathrm{kg} / \mathrm{h}$, via a central line (1). Unfortunately, in our case, central line insertion to allow higher concentrations of potassium chloride was not performed. In our case, we believe the profound hypokalaemia was likely due to severe emesis prior to presentation that severely depleted total body potassium stores. The refractory nature was likely due to ongoing emesis preventing tolerance to first-line oral supplementation, exacerbated by the requirement for insulin administration and correction of metabolic acidosis. Following this case, we have developed local recommendations on the management of hypokalaemia at presentation of DKA, based on evidence and consensus (summarised in Fig. 3). Since implementation of this pathway 6 months ago, cases of refractory hypokalaemia in DKA within our hospital have been effectively managed in a timely manner. We believe that in the absence of

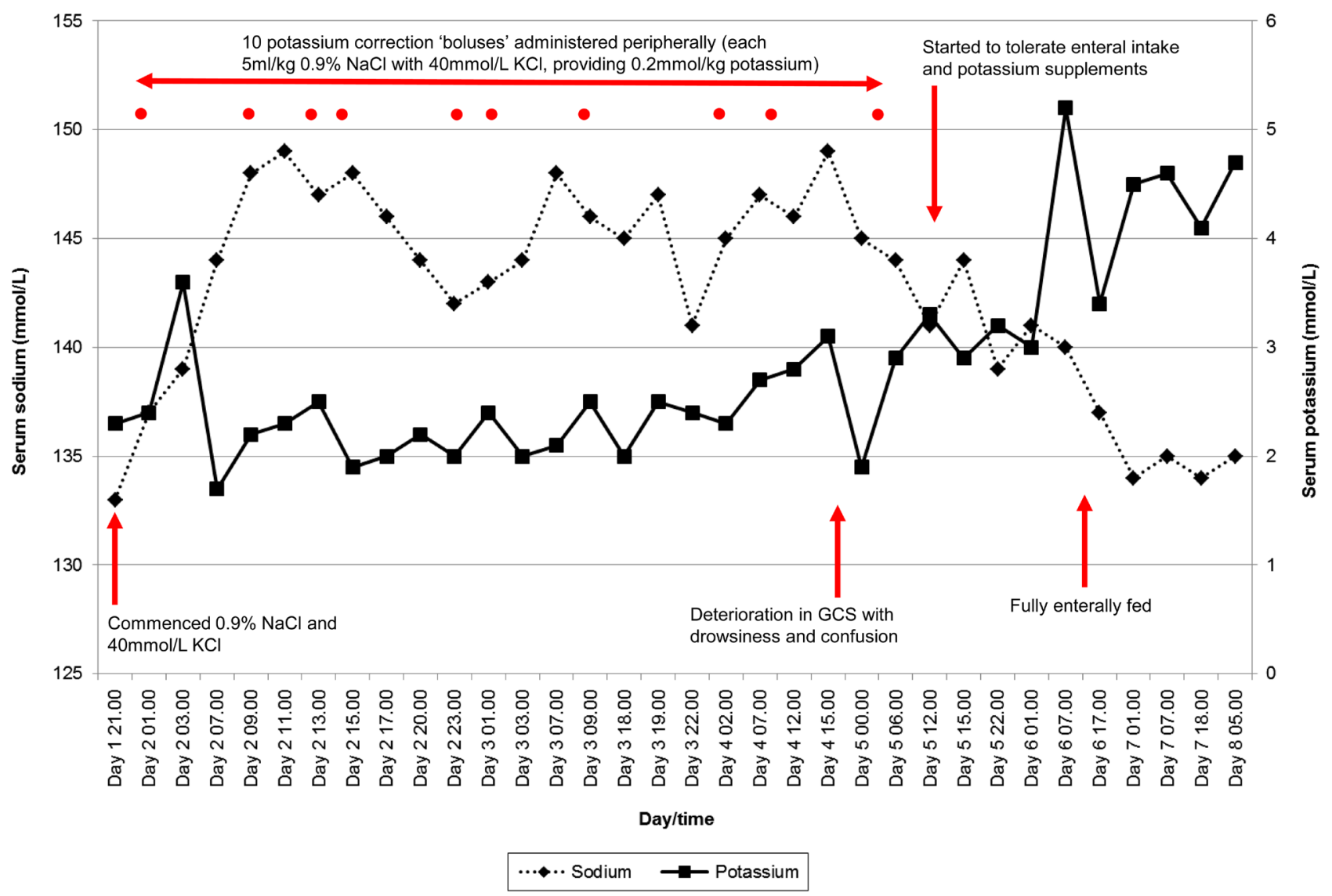

Figure 2

Summary of patient's electrolyte concentration during the first week of her admission, annotated with relevant interventions and onset of neurological deterioration. GCS, Glasgow Coma Score; $\mathrm{KCl}$, potassium chloride; $\mathrm{NaCl}$, sodium chloride. 


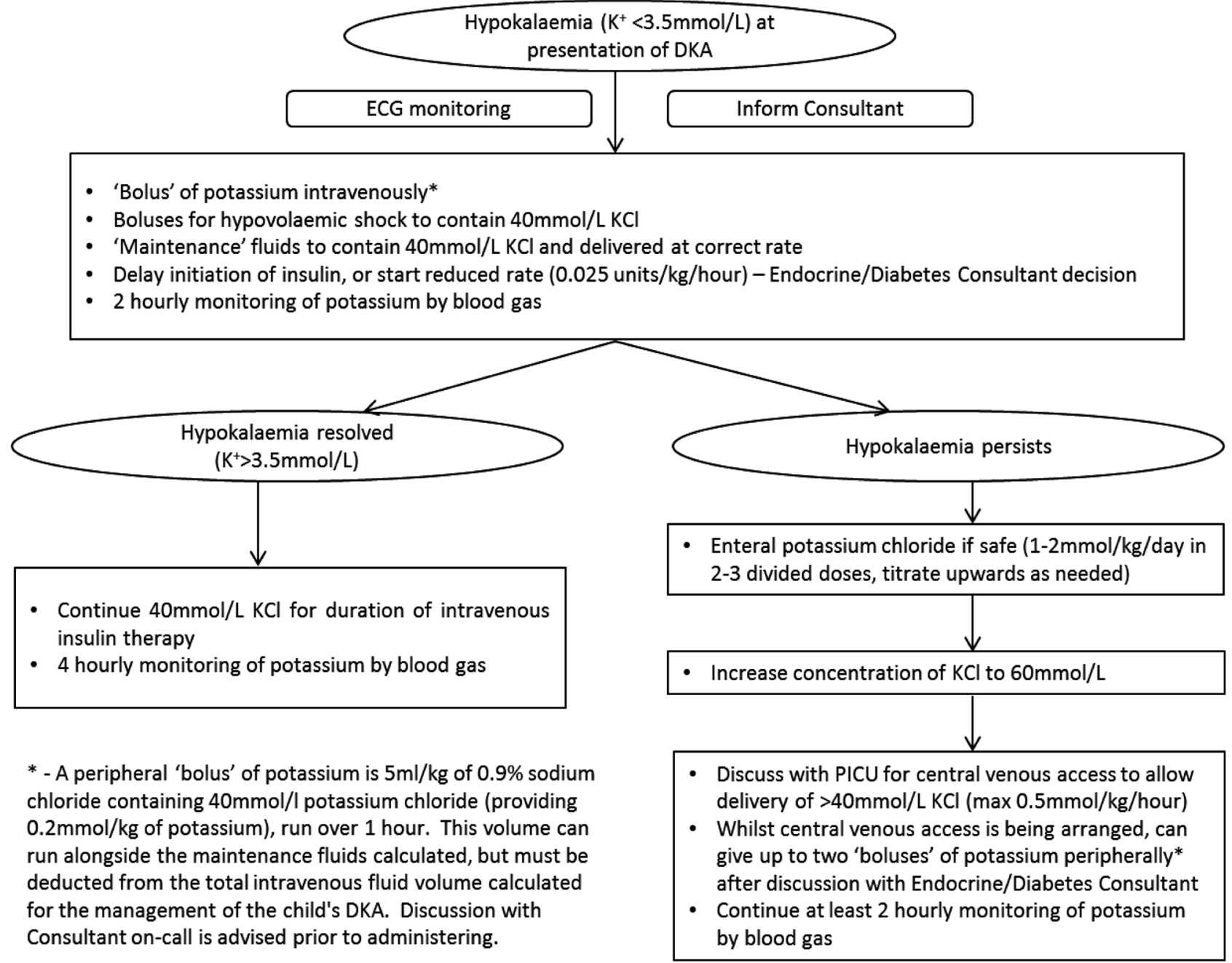

Figure 3

Approach for the management of hypokalaemia existing at presentation of diabetic ketoacidosis. DKA, diabetic ketoacidosis; ECG, electrocardiogram; K+, serum potassium; $\mathrm{KCl}$, potassium chloride; PICU, Paediatric Intensive Care Unit.

good-quality evidence, this pathway represents a safe, rational and practical approach in the management of refractory hypokalaemia in DKA.

CPM is thought to be driven by fluctuations in osmotic forces (4). The condition is rare in children with only approximately 50-60 cases reported in the paediatric literature $(5,6)$, and therefore most of our knowledge comes from adult cases.

Clinical manifestations of CPM depend on the areas affected: acute confusion is usually present, with dysarthria, dysphagia, quadriparesis, spasticity and locked-in syndrome seen with pontine involvement, and movement disorders observed with extrapontine involvement. Definitive diagnosis is made by classical radiological changes, particularly on magnetic resonance imaging. Management is largely supportive and symptomatic. The prognosis in adults varies; a meaningful clinical and functional recovery is expected in approximately $50 \%$ of cases and death in approximately $25 \%$ of cases $(7,8)$. A review of the paediatric literature by Turnbull et al. suggested similar outcomes in children as well: complete or almost complete recovery in 55\% of cases, incomplete or no recovery in $22 \%$ of cases and death in $20 \%$ of cases (6).

The commonest pathogenic factor has been identified to be rapid correction of hyponatraemia causing osmolar shifts $(4,7,8)$. The exact pathophysiology is not known, though hypotheses include osmotic injury to the endothelial cell membrane of the blood-brain barrier resulting in release of myelinotoxic factors or vasogenic oedema and brain dehydration resulting in separation of the axon from its myelin sheath and subsequent 
oligodendrocyte injury (9). Our case presented with serum sodium at the lower end of the normal range, which quickly increased to mild hypernatraemia. Although this is likely to have contributed to the development of CPM, this degree of serum sodium concentration and rate of increase in serum sodium is not uncommon within the setting of paediatric DKA. This is due to hyperglycaemia causing water to move extracellularly from the intracellular compartment, resulting in the reduction of serum sodium concentration by dilution, which is then quickly reversed as the hyperglycaemia corrects, as well as by the intravenous $0.9 \%$ sodium chloride administered as part of the management. Therefore there is likely to be another contributory factor in the development of CPM in this case.

Although hyponatraemia is by far the commonest finding on the presentation of CPM, recently other associations have also been observed - most notably hypokalaemia. A review of 74 adults, who developed CPM with rapid correction of hyponatraemia, identified that $89 \%$ of these cases also had hypokalaemia at presentation (10). Kallakatta et al. observed the characteristics of 25 adult patients who developed CPM, suggesting that hypokalaemia at presentation was prognostic of a poorer outcome (7). Not only has hypokalaemia been associated in cases with hyponatraemia, but also there are reports of CPM developing in normonatraemic individuals in the presence of hypokalaemia $(11,12,13)$. These suggest an independent role for hypokalaemia in the aetiology of CPM.

As well as being very rare in children, CPM is even rarer in the setting of paediatric DKA. To our knowledge, this is only the second reported case, with four other cases developing extra-pontine myelinolysis. The previous cases were mainly associated with serum sodium abnormalities $(5,6)$. Serum potassium abnormalities were either not present or not mentioned. In this case, we believe that her profound and refractory hypokalaemia is likely to be a contributory factor for the development of CPM. This is supported by the adult literature where there is a clear association of CPM with hypokalaemia. The mechanism can only be postulated at present, but is hypothesised to involve sodium-potassium adenosine triphosphatase ( $\mathrm{Na} / \mathrm{K}$-ATPase), an enzyme found in cell membranes that actively transports potassium into cells in exchange for sodium out of cells, and is thus important in the regulation of cell volume. It is known that potassium deficiency results in a downregulation of $\mathrm{Na}$ /K-ATPase concentration in skeletal muscle, cardiac tissue and the brain $(14,15)$, presumably an evolutionary feature to preserve serum potassium concentrations in the event of hypokalaemia. However, this reduced $\mathrm{Na} / \mathrm{K}$ ATPase activity in endothelial cells and oligodendrocytes could result in difficulties preserving cell volume with increasing osmolality, predisposing these cells to injury by lesser fluctuations in osmotic pressures (10), as was seen in our case.

In summary, we have described the case of a 9-yearold girl who presented with DKA and hypokalaemia and sodium at the lower end of the normal range at presentation. While serum sodium increased quickly potentially creating a rapid shift in osmolality, the hypokalaemia remained refractory to treatment, with the development of CPM as an unfortunate complication. This adds to the limited reports of CPM developing in the context of paediatric DKA and suggests a role for profound hypokalaemia in its aetiology. This case also highlights the importance of correcting hypokalaemia early and aggressively in the context of DKA, to avoid devastating complications. We have proposed a management pathway for children presenting with hypokalaemia at presentation of DKA.

\section{Declaration of interest}

The authors declare that there is no conflict of interest that could be perceived as prejudicing the impartiality of the case reported.

\section{Funding}

This report did not receive any specific grant from any funding agency in the public, commercial or not-for-profit sector.

\section{Patient consent}

Written informed consent has been obtained from the patient's guardian for publication of the submitted article and accompanying images.

\section{Author contribution statement}

A Chinoy: writing and revising manuscript, corresponding author; N B Wright: revising manuscript; $\mathrm{M}$ Bone: Physician responsible for the patient, revising manuscript; R Padidela: revising manuscript.

\section{References}

1 Wolfsdorf JI, Allgrove J, Craig ME, Edge J, Glaser N, Jain V, Lee WW, Mungai LN, Rosenbloom AL, Sperling MA, et al. ISPAD Clinical Practice Consensus Guidelines 2014. Diabetic ketoacidosis and hyperglycemic hyperosmolar state. Pediatric Diabetes 201415 (Supplement 20) 154-179. (https://doi.org/10.1111/pedi.12165)

2 Edge JA \& BSPED Clinical Committee. BSPED recommended guideline for the management of children and young people under the age of 18 years with diabetic ketoacidosis 2015. (available at: http://www.bsped.org.uk/clinical/clinical_endorsedguidelines.aspx) 
3 Arora S, Cheng D, Wyler B \& Menchine M. Prevalence of hypokalaemia in ED patients with diabetic ketoacidosis. American Journal of Emergency Medicine 201230 481-484. (https://doi. org/10.1016/j.ajem.2011.01.002)

4 Sterns RH, Riggs JE \& Schochet SS Jr. Osmotic demyelination syndrome following correction of hyponatraemia. New England Journal of Medicine 1986314 1535-1542. (https://doi.org/10.1056/ NEJM198606123142402)

5 Gencpinar P, Tekguc H, Senol AU, Duman O \& Dursun O. Extrapontine myelinolysis in an 18-month-old boy with diabetic ketoacidosis: case report and literature review. Journal of Child Neurology 201429 1548-1553. (https://doi. org/10.1177/0883073813520496)

6 Turnbull J, Lumsden D, Siddiqui A, Lin JP \& Lim M. Osmotic demyelination syndrome associated with hypophosphataemia: 2 cases and a review of literature. Acta Paediatrica 2013102 e164-e168. (https://doi.org/10.1111/apa.12143)

7 Kallakatta RN, Radhakrishnan A, Fayaz RK, Unnikrishnan JP, Kesavadas C \& Sarma SP. Clinical and functional outcome and factors predicting prognosis in osmotic demyelination syndrome (central pontine and/or extrapontine myelinolysis) in 25 patients. Journal of Neurology, Neurosurgery, and Psychiatry 201182 326-331. (https://doi.org/10.1136/jnnp.2009.201764)

8 Singh TD, Fugate JE \& Rabinstein AA. Central pontine and extrapontine myelinolysis: a systematic review. European Journal of Neurology 201421 1443-1450. (https://doi.org/10.1111/ene.12571)
9 Norenberg MD. Central pontine myelinolysis: historical and mechanistic considerations. Metabolic Brain Disease 201025 97-106. (https://doi.org/10.1007/s11011-010-9175-0)

10 Lohr JW. Osmotic demyelination syndrome following correction of hyponatraemia: association with hypokalaemia. American Journal of Medicine 199496 408-413. (https://doi.org/10.1016/00029343(94)90166-X)

11 Mascarenhas JV \& Jude EB. Central pontine myelinolysis: electrolytes and beyond. BMJ Case Reports 20142014 1-3. (https://doi. org/10.1136/bcr-2013-203516)

12 Abdulla MC, Alungal J, Ahammed S \& Narayan R. Central pontine myelinolysis in Sjogren's syndrome with hypokalaemia. International Journal of Rheumatic Diseases 201720 2168-2170. (https://doi. org/10.1111/1756-185X.12847)

13 Sugimoto T, Murata T, Omori M \& Wada Y. Central pontine myelinolysis associated with hypokalaemia in anorexia nervosa. Journal of Neurology, Neurosurgery, and Psychiatry 200374 353-355. (https://doi.org/10.1136/jnnp.74.3.353)

14 Clausen T \& Everts ME. Regulation of the Na,K-pump in skeletal muscle. Kidney International 198935 1-13. (https://doi.org/10.1038/ ki.1989.1)

15 Azuma KK, Hensley CB, Putnam DS \& McDonough AA. Hypokalemia decreases $\mathrm{Na}(+)-\mathrm{K}(+)$-ATPase alpha 2-but not alpha 1-isoform abundance in heart, muscle, and brain. American Journal of Physiology: Cell Physiology 1991260 C958-C964. (https://doi. org/10.1152/ajpcell.1991.260.5.C958)

Received in final form 23 April 2019

Accepted 9 May 2019 